\title{
Antibiotic Prophylaxis in Plastic Surgery Procedures: Indications, Organisms Involved, Medications and Treatment Duration
}

\section{Loonen MPJ ${ }^{1 *}$, De Frene $\mathrm{B}^{2}$, Casaer $\mathrm{B}^{2}$ and Konopka $\mathrm{J}^{3}$}

${ }^{1}$ Department of Plastic and Reconstructive Surgery, Beverly Hills Sunset Surgery Center International (BHSSCI), Valiant Clinic Dubai, United Arab Emirates

${ }^{2}$ Department of Plastic and Reconstructive Surgery, AZ Sint-Jan Brugge-Oostende AV, Belgium ${ }^{3}$ Independent Aesthetic Practice Krakow, Poland

*Corresponding author: Martain Loonen, M.D., Ph.D., FCCP, Consultant Plastic Surgeon and Deputy Medical Director, Beverly Hills Sunset Surgery Center International and Valiant Clinic Dubai, United Arab Emirates, Tel: + 971- 54- 3204135; Email: mloonen@yahoo.com

\section{Review article}

Volume 4 Issue 1

Received Date: May 16, 2020

Published Date: June 26, 2020

DOI: $10.23880 /$ ijtps-16000146

\section{Abstract}

Introduction: Antibiotic prophylaxis has been less extensively studied in plastic surgery and specifications of the ideal duration and types of antibiotics are often not specified.

Very few studies have been carried out looking at optimal use of antibiotic prophylaxis and the practice of antibiotic prophylaxis is largely dependent on physician preference and individual knowledge.

Methods: We have formulated four practical questions relevant for the daily plastic surgery practice and evaluated the scientific evidence to formulate answers to these questions using the medical database of the National Library of Medicine (Pubmed.gov®)

- What are the indications for antibiotic prophylaxis in plastic surgery?

- Which organisms are involved in postoperative plastic surgery infections?

- Which prophylactic antibiotics in which dose should be used?

- What is the duration of prophylactic antibiotic use?

Results: A single dose first-generation cephalosporin such as cefazolin or ampicillin-sulbactam shows the lowest infections rate. Antibiotics are indicated in clean plastic surgery procedures with high-risk factors and in clean-contaminated or contaminated procedures. A short-course administration regimen seemed to be of adequate efficacy and safety.

Conclusion: There is an indication for surgical prophylaxis in clean plastic surgery procedures with high-risk factors and in clean-contaminated or contaminated procedures. A short-course administration regimen seemed to be of adequate efficacy and safety. High-quality prospective trials on larger scale are needed to further confirm these findings.

Keywords: Antibiotics; Prophylaxis; Plastic Surgery; Guidelines

\section{Introduction}

Antibiotic prophylaxis administered perioperatively has been shown to decrease the risk $f$ of post-operative surgical site infection but specifics of use vary widely among different surgical specialties.

Antibiotic prophylaxis was instituted in the 1960's along with the initiation of a wound classification system by the National Research Council in the United Sates [1]. Since its 


\section{International Journal of Transplantation \& Plastic Surgery}

inauguration, extensive clinical research has been carried out in order to develop standards and guidelines for antibiotic type and duration depending on the procedure being performed. Guidelines for the prophylactic use of antibiotics are implemented internationally [2]. Nevertheless, differences in the types of antibiotics and the duration of the prophylaxis treatment are commonly seen between countries and even within the same country.

Antibiotic prophylaxis has been less extensively studied in plastic surgery and specifications of the ideal duration and types of antibiotics are often not specified.

Very few studies have been carried out looking at optimal use of antibiotic prophylaxis and the practice of antibiotic prophylaxis is largely dependent on physician preference and individual knowledge [1].

We have formulated four practical questions relevant for the daily plastic surgery practice and evaluated the scientific evidence to formulate answers to these questions using the medical database of the National Library of Medicine (Pubmed.gov®)

1. What are the indications for antibiotic prophylaxis in plastic surgery?

2. Which organisms are involved in postoperative plastic surgery infections?

3. Which prophylactic antibiotics in which dose should be used?

4. What is the duration of prophylactic antibiotic use?

\section{Indications for Antibiotic Prophylaxis in Plastic Surgery}

Although recommendations for antibiotic prophylaxis exist for cardiac, colorectal, neurosurgical, and orthopedic procedures, national and international guidelines for antibiotic prophylaxis in plastic surgery are lacking. In fact, studies examining the impact of prophylactic antibiotics have produced contradictory results (for example in breast surgery and abdominoplasty surgery).

Most placebo-controlled and retrospective studies for dermatological surgery, abdominoplasty, clean head and neck surgery (radical neck dissection, tumor excision and reconstruction), hand surgery (flexor tendon injuries, carpal tunnel release) and facial procedures (rhinoplasty, facial fractures) have an associated surgical site infection (SSI) rate of $<5 \%$ (clean wound; class I) and prophylactic antibiotics are not indicated [3-6].

An exception is clean breast surgery (augmentation, reduction and reconstructive, lumpectomy, mastectomy, axillary node dissection) and clean plastic surgeries with high-risk factors (for example breast implants) where systemic antibiotic prophylaxis is recommended $[7,8]$.

Oral procedures, such as wedge excision of lip or ear, flaps on the nose and head and neck flaps, have SSI rates of approximately 5-10\% (Class II, clean-contaminated wounds). Antibiotics in class II should be considered [4]. Factors that increase the risk of postoperative infectious complications for plastic surgery procedures include implants, skin irradiation before the procedure, and procedures below the waist. Antibiotics have to be considered in these cases.

Class III (contaminated wounds, mainly hand and head and neck surgery) and Class IV (dirty and infected wounds) should receive antibiotic treatment as antibiotics are therapeutic for these classes $[5,7,8]$.

\section{Organisms Involved in Postoperative Plastic Surgery Infections}

The most common organisms in infections after plastic surgery procedures are Staphylococcus aureus, other staphylococci, and streptococci . Procedures involving macerated, moist environments (e.g., under a panniculus or axilla of an obese individual), below the waist, or in patients with diabetes are associated with a higher rate of infection with gram-negative organisms such as Pseudomonas aeruginosa, Serratia marcescens, or Enterobacteriaceae (including Escherichia coli), Klebsiella species and Proteus mirabilis [9].

\section{Types of Prophylactic Antibiotics and Dose Recommendation}

There is no consensus on the appropriate antimicrobial agent to use for prophylaxis in plastic surgery procedures. Agents with good gram-positive coverage and, depending on the site of surgery, activity against common gram-negative organisms are recommended for patients undergoing clean plastic surgery procedures with risk factors or clean contaminated procedures.

The ideal antibiotic for surgical prophylaxis should (1) cause minimal toxicity or side effects, (2) be effective against the most likely organisms that will cause an SSI but have a narrow spectrum, (3) achieve adequate tissue concentrations at the surgical site for the duration of the procedure, and (4) be administered for the shortest effective period $[10,11]$.

For most patients undergoing plastic surgery procedures, the preferred antimicrobial agent is a first-generation cephalosporin such as cefazolin or ampicillin-sulbactam. 


\section{International Journal of Transplantation \& Plastic Surgery}

An intravenous dose of 1 gram cefazolin is recommended for patients up to 175 pounds (approximately $80 \mathrm{~kg}$ ).

If the patient weighs more than 175 pounds, the dose of cefazolin may be increased to 2 grams intravenously and to 3 grams in case of more than 265 pounds (more than $120 \mathrm{~kg}$ ).

Cefazolin should be administered intravenously 15-60 minutes before the surgery.

An additional dose should be given if the surgical procedure lasts more than three to five hours or if the patient has lost a significant amount of blood (greater than or equal to $1500 \mathrm{~mL}$ ). Patients with a beta-lactam allergy may receive clindamycin or vancomycin.

Clindamycin should be administered intravenously 15-30 minutes before the surgery with a dose of $600 \mathrm{mg}$ IV. Another dose of $600 \mathrm{mg}$ IV should be given in case of surgeries of more than 4 hours.

Due to their longer half-lives, these medications can be redosed at longer intervals if necessary (clindamycin every four to six hours; vancomycin every six to 12 hours). Vancomycin may be given for surgical prophylaxis in facilities with a high incidence of methicillin-resistant Staphylococcus aureus (MRSA) or methicillin-resistant coagulase-negative staphylococci.

Guidelines recommend against the routine administration of vancomycin for antibiotic prophylaxis [1013].

\section{Duration of Antibiotic Treatment}

Antimicrobial prophylaxis should be limited to the shortest duration possible to prevent SSIs (even if a drain or a catheter is left in place or an implant is inserted), limit adverse events, and prevent antimicrobial resistance.

The results of a meta-analysis of 32 studies in head and neck surgery, breast and body surgery and hand surgery were divided into one of four duration categories: no antibiotic prophylaxis, single-dose prophylaxis, 24-hour prophylaxis or extended course (more than 24 hours) prophylaxis. The data was then statistically analyzed for each duration group.

The average percentage of surgical site infections per population for each category was as follows: $14.8 \%$ when no prophylaxis was given, $7.7 \%$ for a single dose prophylaxis, $14.1 \%$ for 24 hours prophylaxis, and $8.8 \%$ for an extended course of antibiotics.

However, as the confidence intervals are wide and overlapping, these findings are not statistically significant. A comparison of the data for antibiotic prophylaxis demonstrates a possible decrease in post-operative incidents for the single dose prophylaxis and the extended course of antibiotics in comparison to no antibiotics and a 24-hour course of antibiotics [14].

\section{Conclusions}

There is an indication for surgical prophylaxis in clean plastic surgery procedures with high-risk factors and in clean-contaminated or contaminated procedures. A shortcourse administration regimen seemed to be of adequate efficacy and safety. High-quality prospective trials on larger scale are needed to further confirm these findings.

\section{Financial Disclosure}

The authors have no financial interest in any of the products, devices, or drugs mentioned in this manuscript.

\section{References}

1. Tadiparthi S (2008) Prophylactic antibiotics for clean, non-implant plastic surgery: what is the evidence? J Wound Care 17(9): 392-394, 396-398.

2. Young B, Ng TM, Teng C, Ang B, Tai HY, et al. (2011) Nonconcordance with surgical site infection prevention guidelines and rates of surgical site infections for general surgical, neurological, and orthopedic procedures. Antimicrob Agents Chemother 55(10): 4659-4663.

3. Babcock MD, Grekin RC (2003) Antibiotic use in dermatologic surgery. Dermatol Clin 21(2): 337-438.

4. Landes G, Harris PG, Lemaine V, Perreault P, Sampalis JS, et al. (2008) Prevention of surgical site infection and appropriateness of antibiotic prescribing habits in plastic surgery. J Plast Reconstr Aesthet Surg 61(11): 1347-1356.

5. Dixon AJ, Dixon MP, Dixon JB (2009) Prospective study of skin surgery in patients with and without known diabetes. Dermatol Surg 35(7): 1035-1040.

6. Casaer B, Tan EK, Depoorter M (2009) The role of antibiotic prophylaxis in abdominoplasty: a review of the infection rate in 300 cases treated without prophylaxis. Plast Reconstr Surg 123(1): 42e.

7. Ariyan S, Martin J, Lal A, Cheng D, Borah GL, et al. (2015) Antibiotic prophylaxis for preventing surgicalsite infection in plastic surgery: an evidence-based consensus conference statement from the American 


\section{International Journal of Transplantation \& Plastic Surgery}

Association of Plastic Surgeons. Plast Reconstr Surg 135(6): 1723-1739.

8. Zhang Y, Dong J, Qiao Y, He J, Wang T, Ma S (2014) Efficacy and safety profile of antibiotic prophylaxis usage in clean and clean-contaminated plastic and reconstructive surgery: a meta-analysis of randomized controlled trials. Ann Plast Surg 72(1): 121-130.

9. Wright TI, Baddour LM, Berbari EF, Roenigk RK, Phillips PK, et al. (2008) Antibiotic prophylaxis in dermatologic surgery: advisory statement 2008. J Am Acad Dermatol 59(3): 464-473.

10. Messingham MJ, Arpey CJ (2005) Updates on the use of antibiotics in cutaneous surgery. Dermatol Surg 31(8): 1068-1078.

11. Gravante G, Caruso R, Araco A, Cervelli V (2008) Infections after plastic procedures: incidences, etiologies, risk factors, and antibiotic prophylaxis. Aesthetic Plast Surg 32(2): 243-251.

12. Bratzler DW, Houck PM (2004) Antimicrobial prophylaxis for surgery: an advisory statement from the National Surgical Infection Prevention Project. Clin Infect Dis 38(12): 1706-1715.

13. American Society of Health-System Pharmacists (1999) ASHP therapeutic guidelines on antimicrobial prophylaxis in Surgery. Am J Health Syst Pharm 56(58): 1839-1888.

14. Richardson C, Workman A, Mattison G, Gupta S (2016) The optimal duration of antibiotic prophylaxis in plastic surgery: A meta-analysis of 32 publications. Health Science Journal 10(2): 1-7. 